\title{
Rare Cause of Abdominal Pain in an Adolescent Patient: Splenic Infarction in Absence of Causative Underlying Hematologic Disorder
}

\author{
Deborah Shimshoni $^{1}$, Chrissy Vandillen ${ }^{2}$ \\ 1. Medicne, University of Central Florida College of Medicine, Orlando, USA 2. Emergency Medicine, St. Cloud Regional \\ Medical Center, St. Cloud, USA
}

Corresponding author: Deborah Shimshoni, deborahshimshoni@knights.ucf.edu

\begin{abstract}
Splenic infarction is a rare medical condition that usually occurs in the setting of hematologic disorders. It is rarely seen in previously healthy adolescents. A wandering spleen increases the risk of splenic infarct due to risk of torsion and is more commonly seen in pregnancy due to increased elasticity of connective tissue. Wandering spleen may also be associated with diseases, such as Ehlers-Danlos, and should be suspected in the patient with possible underlying connective tissue dysfunction. Although rare, splenic infarction must be on the differential for unremitting upper epigastric pain, fever, and vomiting, particularly when patient medical history suggests connective tissue dysfunction. This case discusses the course of a pediatric patient with abdominal pain with complex medical history found to have splenic infarction secondary to torsion of a wandering spleen initially discovered on emergency CT imaging. Although rarely indicated in pediatric patients with abdominal pain, lower threshold for CT imaging for ruling out emergent etiology resulted in life-saving treatment. This case demonstrates the importance of clinical suspicion for emergency etiology of abdominal pain in pediatric patients with medical history suspicious for connective tissue dysfunction, and therefore lowering the threshold for CT imaging to rule out splenic infarction in these patients.
\end{abstract}

Received 06/11/2020 Review began 06/21/2020 Review ended 06/28/2020 Published 07/14/2020

\section{() Copyright 2020}

Shimshoni et al. This is an open access article distributed under the terms of the Creative Commons Attribution License CC-BY 4.0., which permits unrestricted use, distribution, and reproduction in any medium, provided the original author and source are credited.
Categories: Emergency Medicine, Pediatrics, Pediatric Surgery

Keywords: splenic infarcts, child and adolescent, pediatric imaging, emergency medical service, splenic torsion

\section{Introduction}

Splenic infarction is a rare medical condition that is difficult to diagnose in the emergency department. The spleen's extensive vascularity utilizes $5 \%$ of the body's cardiac output, making it susceptible to emboli and thrombosis from hematological disorders [1]. For this reason, splenic infarction more commonly presents in patients with an underlying hypercoagulable state or hematologic disorder such as Gaucher disease or lymphoma [2]. This etiology is rarely ever involved in a previously healthy adolescent patient presenting with abdominal pain. Even in the patient without a hematologic disorder, the possibility for splenic infarction increases if risks are present for splenic torsion. These indications include splenomegaly or wandering spleen. Wandering spleen is the term used to define a spleen that is not adhered to the peritoneum by usual attachments, but instead is attached primarily by a vascular pedicle [3]. This may lead to torsion about the vascular pedicle, cutting off the spleen's blood supply. Most cases of wandering spleen are observed in women of age 20-40 years and may be attributed to multiparity and subsequent increased laxity of connective tissue [3,4]. Wandering spleen is a rare anatomical finding in children and has been associated with Ehlers-Danlos syndrome due to increased laxity of ligaments [5]. These patients with EhlersDanlos associated wandering spleen may eventually succumb to splenic torsion, though such cases are still considered rare and are sparsely available in the current literature $[5,6]$. This case demonstrates a pediatric patient found to have splenic infarction without underlying hematologic causation, but with a possible connective tissue disorder component. This case highlights when to suspect and investigate the possibility of splenic infarction in patients with abdominal pain, nausea, and vomiting.

\section{Case Presentation}

A 16-year-old female presented to a community emergency department with a chief complaint of cramping abdominal pain for two weeks and severe worsening pain with associated nausea and vomiting for two days. She also described sharp pain with inspiration in her left anterior chest. The patient had a medical history that included gastroesophageal reflux disease (GERD), carpal tunnel syndrome, tumor pilomatrixoma of the upper extremity, glaucoma, and splenomegaly of unknown etiology. She denied any recent travel, trauma, or sick contacts. She denied any recent history of sore throat, cough, lightheadedness, leg swelling, diarrhea, hematemesis, hematochezia, or shortness of breath.

She appeared pale with dry mucus membranes and was visibly uncomfortable in pain. Vital signs on exam demonstrated slightly elevated temperature of 99.5 degrees Fahrenheit and tachycardic heart rate of 110 beats per minute. Otherwise, vitals were unremarkable with a blood pressure of $132 / 75 \mathrm{mmHg}$, a respiratory rate of 18 breaths per minute, and oxygen saturation of $99 \%$ on room air. Physical exam revealed diminished 


\section{Cureus}

bowel sounds and diffuse tenderness to palpation with maximal tenderness located in the epigastrium and bilateral upper abdominal quadrants. There was significant splenomegaly, otherwise no hepatomegaly, masses, or peritoneal signs.

Initial labs revealed an elevated white blood cell count of $23.4 \mathrm{~K} / \mu \mathrm{L}$ (reference range 4.1-10.4 K/ $\mu \mathrm{L}$ ), positive anion gap of 20 (reference range 2-12), but a normal lactic acid dehydrogenase (LDH) of $0.9 \mathrm{mmol} / \mathrm{L}$ (reference range 0.4-2 mmol/L). Urine pregnancy test was negative. Due to intractable nausea and continued pain, despite analgesics and anti-nausea medications, CT imaging with contrast was ordered for the abdomen and pelvis. General impression of the CT scan was read by the in-house radiologist as devascularization/infarct of the entire spleen with possible volvulus about the splenic hilum (Figure 1). The patient was immediately transferred via critical care air transport to a tertiary care children's hospital where emergent surgical intervention revealed non-viable splenic torsion secondary to wandering spleen. Surgical removal of the spleen was successful and the patient had a non-complicated recovery.
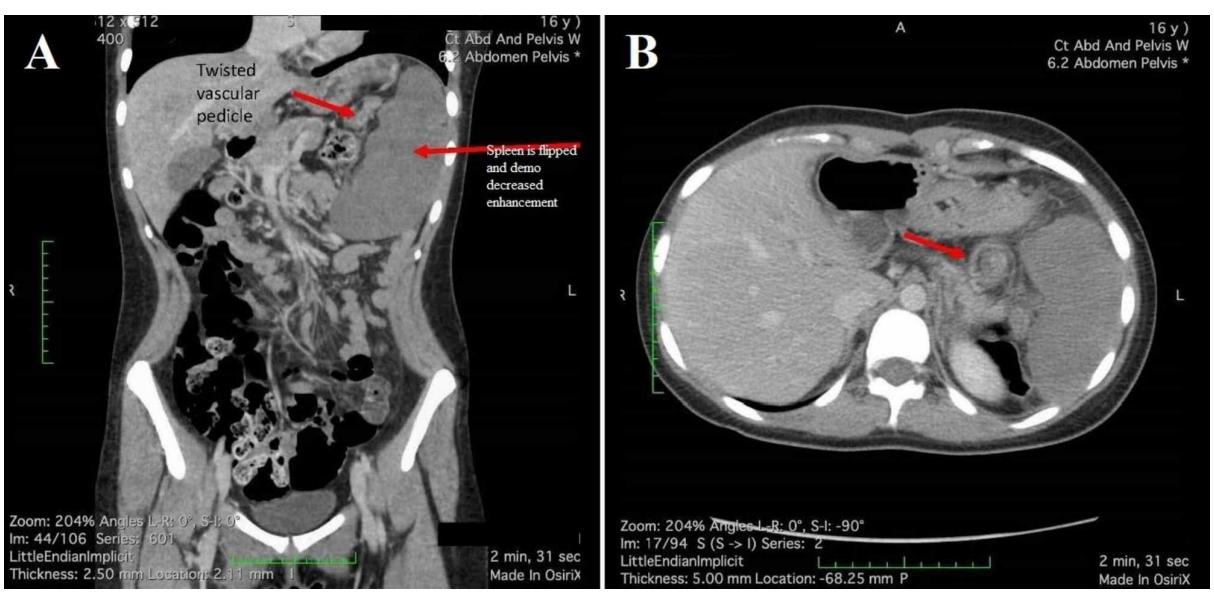

\section{FIGURE 1: CT with contrast}

CT with contrast demonstrating splenic infarct secondary to torsion in a 16-year-old female patient. (A) Sagittal view with noted splenic enlargement and twisted vesicular pedicle. (B) Axial view of splenic infarction.

Post splenectomy, the patient was seen by her primary care physician for chronic fatigue, dysmenorrhea, gum bleeding, and joint hypermobility and was subsequently diagnosed with von Willebrand factor deficiency type 1 . There is now concern that this patient may have Ehlers-Danlos syndrome. The patient had a wandering spleen, an anatomical finding often associated with Ehlers-Danlos syndrome [5]. This along with the patient's congenital glaucoma, GERD, and hypermobility increases the suspicion of Ehlers-Danlos diagnosis although final testing for this diagnosis is still pending [6].

\section{Discussion}

Acute epigastric abdominal pain, nausea, and vomiting with no peritoneal signs, especially in the setting of normal lactate and fever, raises suspicion for gastroenteritis or an infectious etiology in the pediatric patient [7].CT imaging is not generally suggested for this presentation in order to reduce radiation exposure in the pediatric population. However, exceptions are made if suspicion is high for appendicitis, trauma,

pancreatitis, or a palpated mass [7]. If there are continued concerns in patients with abdominal pain such as fever, high white blood cell count, intractable vomiting with continued pain despite medication, CT imaging of the abdomen pelvis should be considered. Pain localized to the left upper quadrant raises suspicion for splenic infarct. In a patient with a complex medical history with a possible connective tissue component, common complaints may reflect a rare underlying etiology.

Splenic infarction may lead to severe consequences if missed on initial emergent exam. Currently, there are limited studies available that attempt to identify the prevalence, etiologies, interventions, and outcomes of splenic infarction in the pediatric population [8]. A massive splenic infarction is described as parenchymal ischemia secondary to vessel occlusion leading to tissue necrosis of at least half of the spleen [9]. For patients below the age of 40 years, etiology is most commonly due to hematologic disease [10]. Other causes include hypercoagulability, trauma, or enlargement secondary to Epstein-Barr viral infection $[2,9,10]$. Many times, an underlying abnormality is found during the workup for the initial presentation of a splenic infarction [2]. Therefore, even if a patient denies having risk factors of splenic infarction, the differential should still be considered in the previously healthy patient with signs and symptoms that may suggest this diagnosis. A study from 2010 suggests that the most likely symptoms found in order of commonality for 
splenic infarction include an elevated LDH, localized left abdominal pain or tenderness, white blood cell count $>12,000$, fever greater than $38^{\circ} \mathrm{C}$, splenomegaly, nausea, and vomiting [2]. Additionally, the presence of a wandering spleen increases the risk of splenic torsion and consequently splenic infarction, a surgical emergency [4]. When the emergency physician is faced with the previously healthy patient with unremitting abdominal pain, splenic infarction should find its way on the differential, particularly in the presence of the aforementioned risk factors or symptoms. This case demonstrates the importance of clinical suspicion to perform a diagnostic procedure for abdominal pain that is not conventionally first line.

\section{Conclusions}

This case demonstrates splenic infarction secondary to splenic torsion in a patient with a chief complaint of nausea, vomiting, and abdominal pain refractory to pharmaceutical intervention found to be tachycardic and febrile on physical exam. Such cases are rare for pediatric patients in the absence of an underlying hematologic disease, and therefore this diagnosis could easily be overlooked due to its vague presentation. This case illustrates the need for emergency physicians to put splenic infarction in the differential diagnosis for patients with intractable vomiting and left upper quadrant abdominal pain. Additionally, this patient's complicated past medical history supports the possibility of an underlying connective tissue disorder, making splenic involvement more likely. In these patients, emergency physicians should have a lower threshold for CT imaging to rule out an emergent etiology for abdominal pain.

\section{Additional Information}

\section{Disclosures}

Human subjects: Consent was obtained by all participants in this study. Conflicts of interest: In compliance with the ICMJE uniform disclosure form, all authors declare the following: Payment/services info: All authors have declared that no financial support was received from any organization for the submitted work. Financial relationships: All authors have declared that they have no financial relationships at present or within the previous three years with any organizations that might have an interest in the submitted work. Other relationships: All authors have declared that there are no other relationships or activities that could appear to have influenced the submitted work.

\section{References}

1. Schattner A, Adi M, Kitroser E, Klepfish A: Acute splenic infarction at an academic general hospital over 10 years: presentation, etiology, and outcome. Medicine. 2015, 94:1363. 10.1097/md.0000000000001363

2. Chapman J, Helm TA, Kahwaji CI: Splenic infarcts. StatPearls Publishing, Treasure Island, FL; 2020.

3. Desai DC, Hebra A, Davidoff AM, Schnaufer L: Wandering spleen: a challenging diagnosis. South Med J. 1997, 90:439-443. 10.1097/00007611-199704000-00017

4. Brown CV, Virgilio GR, Vazquez WD: Wandering spleen and its complications in children: a case series and review of the literature. J Pediatr Surg. 2003, 38:1676-1679. 10.1016/S0022-3468(03)00582-7

5. Rattay T, Shrivastava A, Higman DJ, Francombe J: Spontaneously ruptured splenic aneurysm in a young patient with Ehlers-Danlos syndrome. BMJ Case Rep. 2011, 2011:bcr0120113753. 10.1136/bcr.01.2011.3753

6. De Paepe A, Malfait F: The Ehlers-Danlos syndrome, a disorder with many faces . Clin Genet. 2012, 82:1-11. 10.1111/j.1399-0004.2012.01858.x

7. Reuchlin-Vroklage LM, Bierma-Zeinstra S, Benninga MA, Berger MY: Diagnostic value of abdominal radiography in constipated children: a systematic review. Arch Pediatr Adolesc Med. 2005, 159:671-678. 10.1001/archpedi.159.7.671

8. Vijayaraghavan S, Thomas J: Clinical spectrum of splenic infarction: a South Indian perspective . Int Arch Med. 2016, 9:1-5. 10.3823/1997

9. Mortazavi AR, Joudi M, Zabolinejad N, Aelami MH, Farhangi H, Alamdaran SA: Massive splenic infarction with specific sonographic feature: two case reports with rare etiologies. J Clin Gastroenterol Treat. 2018, 4:058. 10.23937/2469-584X/1510058

10. Bhattarai P, Pierr L, Adeyinka A, Sadanandan S: Splenic infarct: a rare presentation in a pediatric patient . JNMA J Nepal Med Assoc. 2014, 52:1017-1019. 10.31729/jnma.2805 